\title{
Quantum Gravity and Mereology: Not So Simple
}

\author{
Sam Baron and Baptiste Le Bihan \\ forthcoming in \\ the Philosophical Quarterly
}

\begin{abstract}
A number of philosophers have argued in favour of extended simples on the grounds that they are needed by fundamental physics. The arguments typically appeal to theories of quantum gravity. To date, the argument in favour of extended simples has ignored the fact that the very existence of spacetime is put under pressure by quantum gravity. We thus consider the case for extended simples in the context of different views on the existence of spacetime. We show that the case for extended simples based on physics is far more complex than has been previously thought. We present and then map this complexity, in order to present a much more textured picture of the argument for extended simples.
\end{abstract}

Keywords - quantum gravity; extended simples; spacetime; realism; eliminativism; mereology 


\section{Introduction}

A number of philosophers have argued in favour of extended simples on the grounds that they are needed by physics. The arguments typically appeal to promising research approaches aimed at the development of new fundamental physical theories. For instance, the physicist Brian Greene writes that according to the leading research program in fundamental physics, namely superstring theory, the world must be regarded as being made of fundamental, onedimensional entities - strings — which are both extended entities and lacking proper parts:

[S]trings are truly fundamental - they are "atoms," uncuttable constituents, in the truest sense of the ancient Greeks... From this perspective, even though strings have spatial extent, the question of their composition is without any content. (Greene 1999: 141-2)

Along these lines, Craig Callender writes (see also McDaniel 2007b; 2007a: 235):

On its most natural interpretation, superstring theory... posits extended simples. I say 'most natural' because the theory was initially motivated by the fact that the topology of interacting continuous one-dimensional extended entities avoided the ultraviolet divergences that plagued graviton-graviton scattering. The onedimensionality of strings really is a significant part of the original attraction of the theory. (Callender 2011: 38-9)

Rettler writes that both string theory and loop quantum gravity (another popular approach to fundamental physics) entail the existence of extended simples:

Both theories entail the existence of extended simples. If string theory is true, then there are extended simples, because strings are extended (one-dimensional) simples. If loop quantum gravity is true, then space is discrete; so, its smallest 
parts are extended. If an extended simple fills up some but not all of an extended region, then there's a part of the region it fills up; and so there are regions smaller than it. But that's impossible. So, if loop quantum gravity is true, the smallest things are also extended. (Rettler 2019: 851)

Rettler goes on to claim that string theory and loop quantum gravity are the only two approaches to unifying general relativity and quantum physics to produce a theory of quantum gravity (QG) and so the case for extended simples is decisive. While Rettler is incorrect about the number of available options - there are many more approaches to QG than the two he considers - we will restrict our focus to string theory and loop quantum gravity as well, since these are the two leading approaches to QG in terms of the number of involved physicists. $^{1}$

Thus the question we are interested in is whether philosophers and physicists are right that string theory or loop quantum gravity entail the existence of extended simples of some kind. Answering this question is not straightforward however as those approaches appear to imply that the world lacks spatiotemporal structure. ${ }^{2}$ Of course, there are many entities that do not appear in fundamental physics that we nonetheless adopt a realist attitude toward. A central question for these approaches, then, is what to say about spacetime. Does spacetime exist, or is its absence in fundamental physics indicative of its absence tout court?

We won’t try to settle this hard question here. Nor do we intend to argue decisively for or against the existence of extended simples. Rather, because a discussion of the connection between the status of spacetime and the problem of extended simples is presently lacking in the literature, we aim to correct this situation. Our goal is to show that the case for

\footnotetext{
${ }^{1}$ For a short presentation and discussion of the other views, see e.g. Huggett \& Wüthrich (2013).

${ }^{2}$ For further discussion of exactly how much spatiotemporal structure is lost see Huggett \& Wüthrich (2013) and Le Bihan \& Linnemann (2019).
} 
extended simples based on physics is far more complex than has been previously thought. We aim to map this complexity, in order to present a much more textured picture of the case for extended simples from physics.

We show that whether a given approach to QG entails the existence of extended simples depends heavily on the existence of spacetime, but not only. It also depends on how spacetime exists. Whether physics recommends extended simples thus highly depends upon a particular metaphysical interpretation of the physical situation at issue. A look ahead: we begin, in $\S 2$, by providing a brief overview of string theory and loop quantum gravity. In $\S 3$, we introduce two broad arguments in favour of extended simples based on physics. In $\S 4$, we present four distinct metaphysical interpretations of QG which disagree over whether and how spacetime exists. We go on to show which of these interpretations facilitate an argument in favour of extended simples from physics and which do not. Along the way, we sketch out some examples of metaphysical approaches that warrant the conclusion that extended simples exist but we do not defend these approaches. We simply highlight the kind of metaphysical assumptions needed if the case for extended simples based on physics is to be made.

\section{Two Approaches}

Quantum gravity is the name of a cluster of research programs in physics aimed at the formulation of a new, more fundamental theory, weaving together everything we learned from our two most fundamental theories in physics at the moment: general relativity (GR) and the standard model of particle physics, which is formulated in the framework of quantum field theory. It is well known that the two frameworks are in tension, if not inconsistent, as it seems impossible - or at the very least tremendously complicated - to quantize gravity in the same way as the other forces (electromagnetism, the weak force and the strong force). 
More precisely, it seems impossible to quantize gravity all the way down for all energy scales. Physicists therefore aim at formulating a new, more fundamental, theory with the resources to describe reality for all scales — a theory said to be 'ultra violet complete' or 'UV complete' as the amount of energy attributed to field fluctuations corresponds to frequencies higher than the range of violet light. While it is debatable as to whether a theory of quantum gravity should necessarily be UV complete - as Crowther \& Linnemann (2019) argue, UV completion is more akin to a guiding principle than to a necessary constraint on any theory of quantum gravity - there exists various research programs at different levels of maturity aiming at such a UV complete theory. The most developed and promising approaches at the moment are string theory and loop quantum gravity (henceforth, LQG).

String theory is the most popular approach to quantum gravity (again, in terms of the number of physicist involved) and is often presented as a prospective final theory of everything (see e.g. Greene 1999 and Dawid 2013 for accessible introductions). The view describes a world made of one-dimensional entities: strings. Those strings are incredibly tiny and rigid and may oscillate with various frequencies, giving rise to phenomena that we categorize as the particles and fields in our less fundamental theories: GR and quantum field theories. String theory, in a sense, starts from the standard model of particles and seeks to recover gravity via the view that gravitational interactions are mediated by a particle: the graviton, which is then reduced to a particular state of a string (more precisely, to a closed string with a low-energy vibrational state).

String theory aims at the unification of the various field theories by adding six spatial dimensions to the spacetime manifold (Polchinski 1998). Since the so-called second superstring revolution, this 'standard' picture of strings living in a ten-dimensional spacetime is regarded as a mere approximation of a more fundamental structure to be described by a 
more fundamental theory, the so-called 'M-theory' (conjectured to be eleven-dimensional). ${ }^{3}$

Indeed, 'string theory' is in fact a paradigm made of five string theories with strange relations of duality existing between sub-classes of solutions to those theories. As the solutions are empirically equivalent, they are taken to describe the very same facts and to be dual descriptions of the same physics. ${ }^{4}$ One especially important sort of duality is T-duality: different models with different spatial dimensions (i.e. compactification radii of the six added dimensions) are physically equivalent (Strominger et al. 1996). A prominent philosophical interpretation of duality among philosophers of physics is the common core view which states that the ontology of the world is captured by the common core structure of the different string theories (cf. Huggett 2017) - hopefully to be obtained one day with the M-theory. It is notoriously challenging to make sense of this common core though as it is lacking both a mathematical and an ontological description - but it seems that it will not include space. This is due to the fact that T-duality holds between string theories that have different spaces associated with different compactification radii, and so the common core view may excise space as a superfluous mathematical artefact. Although, there do exist other ontological interpretations of duality (for a review see Le Bihan and Read 2018), they all assume that we have no reason to identify GR spacetime with one of the dual background structures. On top of that, a particular approach to string theory, namely the worldsheet view, entails that string theory employs a two-dimensional and scale-invariant structure, prompting again a question about the existence of a (four-dimensional and not scale invariant) spacetime. ${ }^{5}$ As a result, it is highly non-trivial to link spacetime as described by our most fundamental theory of spacetime at the moment - namely, GR - to a structure that is both more fundamental and

\footnotetext{
${ }^{3}$ Hořava \& Witten (1996).

${ }^{4}$ See Huggett \& Wüthrich (2013: 280), Huggett (2017) and Butterfield (forthcoming).

${ }^{5}$ See Witten (1996) and Huggett, Vistarini \& Wüthrich (2013: section 3), Huggett \& Vistarini (2015), Baker (2016: section 4.2) and Le Bihan (2020) for philosophical discussion.
} 
potentially non-spatial.

LQG takes a different approach to QG (for an introduction, see Rovelli 2004; Rovelli \& Vidotto 2014). Rather than starting from the standard model of particles, and, from there, seeking to accommodate gravity, LQG starts with GR and thus with gravity and aims to provide a quantum account of the gravitational field, with the hope of reconciling it with the quantum fields of the standard model down the track. In the kinematical form of the theory, the fundamental posits of the theory are represented by networks of intersecting loops called 'spin networks'. Spin-networks can be represented by undirected graphs: a structure of nodes connected by edges. Exactly what the nodes and edges represent is controversial. On one way of interpreting the theory, each node in a spin-network represents a 2D spatial surface, and every edge in a spin-network represents a 3D spatial volume. A group of nodes and edges can thus represent an atom of space (in something like the way that a cube can be represented as a two-dimensional net, like a Latin cross). The entire spin-network structure therefore represents a structure of spatial atoms, connected to one another by relations of adjacency as encoded by the graph-theoretic representation.

The kinematical form of the theory ultimately gives way to a dynamical version of the theory, and so it is the dynamical form that we will focus on here (everything we say applies to the kinematical form too). In the dynamical form of LQG, spin-networks are replaced by spin-foams. A spin-foam is, very roughly, an extrusion of the spin-network structure through a higher dimension. As with the spin-network structure, it is controversial as to exactly how spin-foams should be physically interpreted. One way of interpreting spin-foams, however, is to treat the spin-foam structure as representing a lattice of spatiotemporal atoms.

As usual in quantum theories, many fundamental classical quantities are substituted by operators in LQG giving, as output, discrete values. Importantly, for our purpose, areas and volumes are treated as operators, entailing that volumes and areas can only take discrete 
values - thereby offering, or so it seems, reason to interpret LQG as relying on an ontology of discrete entities.

There is an important conceptual gap between GR and LQG. GR is continuous and classical, while the fundamental structure of LQG, with its quantum operators, appears to be discrete and quantum. This conceptual gap is widened by another intriguing feature of LQG, namely disordered locality (see e.g. Markopolou \& Smolin 2007 and Huggett \& Wüthrich 2013: 279-80). In some models of LQG, there is a deviation between the ordering of GR events, and the ordering of the LQG entities that correspond to those events. While, in LQG, two entities might be local to one another in a spin-network (i.e. they are located at adjacent nodes in the spin-network structure) those entities may correspond to events in spacetime that are arbitrarily far away from one another. Disordered locality signals that GR spacetime differs substantially from the spin-foam structure and, thereby, that one should not be too quick in identifying the fundamental LQG structure with spacetime itself. Indeed, according to Lam \& Wüthrich (2018: 49) the differences between the spin-foam structure and spacetime warrant an even stronger conclusion: namely, that any such identification would be a mistake. We cannot interpret spin-foams in standard spatiotemporal terms. This is one of the principal reasons why interpreting a spin-foam as a lattice of spatiotemporal atoms is controversial. The discrete entities that constitute the lattice structure of a spin-foam may not be spatiotemporal at all.

There is a lot more to be said regarding LQG and string theory, but our aim is not to provide an exhaustive discussion of the views here. The central take-home message is that there is no consensus on how to interpret either theory. In particular, there is no agreement on whether string theory and LQG are spacetime theories: theories that posit some spatiotemporal structure. As we shall see, the fact that this interpretative question remains open rather complicates the case for extended simples. Before we explain why, let 
us take a brief look at the arguments for extended simples that have been offered to date.

\section{Two Arguments}

In this section, we will outline two arguments aimed at establishing the existence of extended simples based on the approaches to physics described above. In the first instance, we will simply outline the arguments without focusing too much on the physics. After that, we will discuss the support for the arguments provided by particular theories. Note that we are not defending these arguments, nor do we claim to have invented them. They are supposed to be faithful reconstructions of arguments offered by philosophers and physicists elsewhere.

Let us begin by saying a bit more about what extended simples are. In order to do this, we will work within a locative framework - a metaphysical framework, that is, which presumes a primitive location relation. The primitive location relation that we will use is a notion of exact location: $L(x, y)$. Gilmore (2018) provides the following gloss on exact location:

An entity $x$ is exactly located at a region $y$ if and only if $x$ has (or has-at- $y$ ) exactly the same shape and size as $y$ and stands (or stands-at-y) in all the same spatial or spatiotemporal relations to other entities as does $y$.

In addition to a notion of location, we will assume the following broad picture of spacetime. Spacetime is anything that can be faithfully represented by a smooth, differentiable manifold equipped with a Lorentzian metric $\langle M, g\rangle$. Spacetime, we will assume, has spacetime regions as parts. A spatiotemporal region is extended when the metric distance between any two points in the manifold representation of the region is non-zero.

Using exact location and the notion of a spacetime region, we can define two varieties of extended simple. First: extended simple material objects (cf. McDaniel 2007a).

[ESO] $x$ is an extended simple material object $=_{d f} x$ is exactly located at an 
extended spatiotemporal region $r$ and $x$ has no proper parts.

Second: extended simple spatiotemporal regions.

$[\mathbf{E S R}] x$ is an extended simple spatiotemporal region $=_{d f} x$ is an extended spatiotemporal region $r$ and $x$ has no proper parts.

Note that we will set aside specific issues pertaining to the temporal extension of extended simples. We will thus think of extended simple material objects as spatially extended simples, leaving unspecified whether these are also temporally extended simples (in the terminology used by McDaniel 2007a: 134, 'spanners'), since this matter relies on complex views about the way entities persist through time. By contrast we will treat extended simple spatiotemporal regions as spatiotemporally extended since there is no analogous reason to be shy about treating space and time as two abstractions from a more fundamental entity, namely spacetime. At any rate, we will have reason to revisit these definitions later on. For now, however, they are sufficient to formulate the two central arguments in favour of extended simples that have been informally offered in the literature to date.

Broadly speaking, there are two ways to argue from a physical theory to the existence of extended simples. The first, and most direct way, is to argue that a given physical theory $T$ posits some entity $x$ that is both extended and mereologically simple, and so a realist commitment to that theory is sufficient for an ontological commitment to extended simples.

Call this: the simple argument for extended simples. There are two outcomes of the simple argument, depending on what $x$ might be. If $x$ is a material object, then the simple argument establishes an implication from a given theory to the existence of extended simple material objects. Call this: the simple material argument. If, by contrast, $x$ is a region of spacetime, then the simple argument establishes an implication from a given theory to the existence of extended simple spatiotemporal regions. Call this: the simple region argument. 
The second, more indirect method of arguing for extended simples applies when a theory does not directly posit an entity $x$ that is mereologically simple and extended and so realism about the theory alone is insufficient to warrant a commitment to extended simples. A case is then made for extended simples by arguing that we can infer the simplicity of some entity posited by the theory at hand by combining realism about the theory in question with one or more plausible principles that go beyond the content of that physical theory. The central argument along these lines is the harmony argument.

The harmony argument uses a principle of mereological harmony, namely Principle 5 from Saucedo (2011: 227) (the original principle is formulated in terms of parts and sub-regions; we have modified the principle slightly to focus on proper parts and proper sub-regions):

(H) $x$ is a proper part of $y$ iff $x$ 's location is a proper sub-region of $y$ 's location.

Using this principle it is possible to argue from discreteness about spacetime to the existence of both simple objects and simple regions. We must be careful, however, to differentiate between two notions of discreteness that might be applied to spacetime. First, there is a topological notion. Spacetime is discrete in this sense when there are spacetime points that are isolated - i.e. there is a neighbourhood around each point that contains no other spacetime points - and spacetime is constituted by these points. Second, there is a regionbased notion of discreteness. Spacetime is discrete in this sense when there are no spacetime points but, instead, there are extended spacetime regions that have no proper sub-regions and it is these minimal regions that constitute spacetime. ${ }^{6}$

Call the first notion of discreteness 'discreteness ${ }_{T}$ ' and the second notion of discreteness 'discreteness ${ }_{R}$ '. We reserve the term 'discrete' as a generic way of referring to discrete structures of any kind. If spacetime is $\operatorname{discrete}_{T}$, then the case for extended simple regions is dead on arrival as all extended regions have proper parts, namely the points that consti-

\footnotetext{
${ }^{6}$ We are grateful to two referees of this journal for drawing our attention to this distinction.
} 
tute them. More promising for the friend of extended simples is the possibility of finding evidence of discreteness ${ }_{R}$ in physics. Indeed, if spacetime is discrete $_{R}$, then there are some spatiotemporal regions that have no proper spatiotemporal sub-regions. For, if they did, those sub-regions would per impossible be smaller in size than their parent regions. From there, it is possible to argue for simple objects using $(H)$ as follows (where ' $C$ ' is the proper sub-region relation):

1. There exists at least one material object $e$ and a minimal extended spatiotemporal region $r$ such that Ler.

2. $e$ has a proper part $p$ only if there exists a region $r *$ such that $r * \subset r$ and Lpr*.

3. If $r$ is a minimal spatiotemporal region then there does not exist a spatiotemporal region $r *$ such that $r * \subset r$ and $L p r *$.

$\left(\right.$ Discreteness $\left._{R}\right)$

4. e has no proper parts.

5. $e$ is an extended simple material object.

Something like this argument is proposed by Braddon-Mitchell and Miller (2006), except they don't frame the view in terms of $(\mathrm{H})$. Instead, they appeal to a similar principle of occupation drawn from Markosian (2014). We assume, however, that one can translate location talk into occupation talk and vice versa and so there's no substantive difference between the two arguments. The argument for simple regions is similar to the object argument above:

1. There exists at least one minimal extended spatiotemporal region $r$.

2. $r$ has a proper part $p$ only if there exists a region $r *$ such that $r * \subset r$.

3. If $r$ is a minimal spatiotemporal region then there does not exist a spatiotemporal region $r *$ such that $r * \subset r$.

$\left(\right.$ Discreteness $_{R}$ )

4. $r$ has no proper parts. 
5. $r$ is an extended simple spatiotemporal region.

As we will see in a moment, it is unclear whether spacetime really is $\operatorname{discrete}_{R}$ in the approaches to QG we consider and, thereby, whether the harmony argument ultimately succeeds.

\section{Four Interpretations}

The two arguments outlined above are standardly aimed at theories of QG like LQG and string theory. Without a particular interpretation of these theories, however, it is impossible to determine the fate of extended simples. The trouble is that, as indicated in $\S 2$, it is unclear what the status of spacetime is in these theories. This is perhaps clearest in the case of LQG, where the basic posits of the theory - spin-networks in the canonical approach, or spin-foam in the covariant one - do not seem to stand in any spatiotemporal metric relations to one another. Extended simples, however, presuppose a viable notion of spatiotemporal extension. It is thus necessary for determining whether physics implies extended simples that we first determine the status of spacetime in the context of QG.

Whether or not spacetime exists according to QG is an ongoing problem in both physics and philosophy. ${ }^{7}$ We cannot hope to settle this hard problem here. What we can do, however, is consider a range of different metaphysical interpretations of QG and consider the case for extended simples against the backdrop of this interpretive framework. Our framework is thus based on four questions. First, for a given approach to QG does spacetime exist? Second, if spacetime exists, is it continuous or discrete? Third, assuming that spacetime exists, are there, in addition, non-spatiotemporal concrete entities? And, fourth, assuming that

\footnotetext{
${ }^{7}$ This problem is typically described in physics and philosophy of physics as the problem of interpreting the 'emergence' of spacetime. See Crowther (2018) and Wüthrich (2017: 328) for discussion.
} 
spacetime exists and there are non-spatiotemporal concrete entities, what is the relationship between them?

We can then differentiate, very broadly, between four distinct metaphysical views: ${ }^{8}$ three versions of realism and eliminativism. The eliminativist states that spacetime does not exist according to the theory of QG. In fact, they maintain, spacetime does not exist full-stop. Rather, spacetime is to be straightforwardly eliminated in favour of the new ontology given by the theory of QG. Thus, on this view, only non-spatiotemporal entities exist (Baron 2020 comes close to defending this view). The full-blown realist, by contrast, maintains that there exists only spatiotemporal entities. She thus maintains that spacetime is, or at the very least could in principle be, identified with some basic structure within a theory of QG, and everything in QG is then located within this structure.

The next two options agree with the full-blown realist that spacetime exists, but disagree about the status of non-spatiotemporal entities. According to these further realist options, there exist both spatiotemporal and non-spatiotemporal entities. In both cases, spacetime depends, for its existence, on the non-spatiotemporal entities at issue.

Spacetime is thus, in some sense, non-fundamental, in virtue of its dependence on nonspatiotemporal entities. The third metaphysical interpretation of QG takes this fundamentality talk very seriously indeed. According to derivative spacetime realism spacetime is real and grounded in a more basic non-spatiotemporal structure (see e.g Wüthrich 2017: 298 for expressing claims in that direction). Spacetime thus depends, on this picture, on a nonspatiotemporal reality, which primitively grounds spacetime.

The fourth and final option is mereological spacetime realism. According to this view, spacetime is real and composed from basic non-spatiotemporal building blocks (Le Bihan 2018). At first, mereological spacetime realism may not seem all that different from derivative space-

\footnotetext{
${ }^{8}$ Three of the views considered are featured in the taxonomy provided by Le Bihan (2018).
} 
time realism. Both views accept that spacetime depends on non-spatiotemporal entities in some sense. The two views are not the same, however. The grounding relation in derivative spacetime realism, as noted, is primitive: it is not to be further understood in mereological terms. One might respond that mereological connections nonetheless imply the existence of primitive grounding ones. Certainly, for some, the whole is grounded in its parts. Such a view, however, is not universally accepted, and so we won't assume it here.

For each of the three versions of realism introduced above, we can make a further distinction based on whether existing spacetime is taken to be continuous or discrete. There are, thus, really six realist views on the table (nine once we factor in the distinction between $\operatorname{discreteness}_{T}$ and $\operatorname{discreteness}_{R}$ ). To simplify matters, however, we won't name each of the six alternatives. Nonetheless, we will consider all six views in what follows. Our discussion of the discrete and continuous forms of each view will, however, be rolled into our more general discussion of extended simples.

Note that while we have stated the four metaphysical interpretations of QG in general terms, we recognize that different interpretations may be more or less appropriate for a given approach to QG. It depends a lot on what the physics appears to license in any given case. We are therefore careful to be sensitive to this fact as we consider the interpretations in detail below.

Note also that while we take it to be an open question as to how, exactly, we should metaphysically interpret QG, we admit that a kind of realism about spacetime is likely to be the more intuitive view for many and has been defended with different flavours (see e.g. Lam \& Wüthrich 2018; forthcoming and Yates forthcoming). Nonetheless, spacetime eliminativism remains a possible option and so it is worth considering. We will therefore begin with the eliminativist view and discuss it briefly, before moving on to realist alternatives. Unsurprisingly, eliminativism undermines the case for extended simples. What is perhaps more 
surprising is that adopting realism — starting with full-blown realism — does not straightforwardly help the case for extended simples to succeed.

\subsection{Spacetime Eliminativism}

Spacetime eliminativism is a radical view, and so it is worth saying a bit more about it. Very roughly, the eliminativist picture treats the relationship between QG and GR as analogous to the relationship between GR and Newtonian mechanics. The mathematical structure of Newton's mechanics is derivable from GR (as described by a Newton-Cartan theory, namely a geometrical reformulation and generalisation of the original Newtonian theory) under three conditions: when velocity tends toward zero, when the gravitational field is weak and when the gravitational field is static. But despite the fact that the mathematics of Newton's theory are derivable from GR in a certain limit, through a certain reformulation, it is generally assumed that the ontology of Newtonian physics is not preserved in the new relativistic paradigm. More specifically for our concern, an anti-realist stance toward space and time, understood in a pre-relativistic way as two globally separated manifolds, is generally assumed in favour of a realist stance toward GR spacetime.

Similarly, we can imagine that despite being derivable from QG in a specific regime (namely a range of energy), the background structure of GR, as described by the metric field, could be eliminated in favour of some structure of the more fundamental theory. In the case of LQG, for instance, this may mean eliminating spacetime in favour of the spinnetwork (or spin-foam) structure, which is then interpreted as being non-spatiotemporal. Despite being eliminated, we may say that the spin-network approximates spacetime in the sense described by Butterfield \& Isham (1999): by neglecting high energy, the spin-network structure behaves like a spatiotemporal one. Again, this is analogous to the Newtonian case: spacetime approximates pre-relativistic space and time under certain conditions. 
According to spacetime eliminativism, then, spacetime does not exist at all. Rather, what exists is some non-spatiotemporal structure. Spacetime eliminativism undermines the case for extended simples entirely, in the manner suggested at the start of the previous section. If spacetime does not exist, then nothing is extended and so a fortiori no simple is extended.

Is there a way to rebuild the case for extended simples in the face of spacetime eliminativism? Here is a speculative suggestion. It may be that while spacetime does not exist, there exists some non-spatiotemporal structure that permits a coherent notion of location and extension. If so, and if the structure is discrete then it is conceivable that the case for extended simples can be revived. We would, of course, need to redefine extended simples in non-spatiotemporal terms. But perhaps indeed this can be done. In the absence of an account of non-spatiotemporal extension, however, we will set aside the eliminativist view and press on to the realist options, starting with full-blown realism.

\section{$4.2 \quad$ Full-Blown Realism}

According to full-blown realism, spacetime exists, and there are no non-spatiotemporal concrete entities at all: everything is spatiotemporally located. Spacetime thus exists at all levels of description including the most fundamental levels to be described by a theory of QG. Full-blown realism associates a much more standard picture of reality with physics, and so it might be thought that it provides the greatest scope for establishing the existence of extended simples. At the very least, we have the basic spatiotemporal structure needed to make sense of extension. To see whether the prospects for extended simples are indeed improved under full-blown realism, let us apply each of the arguments introduced in $\S 2$, starting with the simple material argument and with string theory.

The material posits of string theory are strings. So for the simple material argument to work, realism about string theory and thus strings must imply the existence of extended 
simples. Baker (2016) has already criticized this argument in the case of string theory and so we will be brief. Baker provides compelling reasons to suppose that strings do, in fact, have parts: they can be split and joined. Splitting and joining is what we would expect of composite objects - but certainly not of simples. He goes on to argue that any strings can be split, and that all strings split into further strings (Baker 2016: 6). Assuming that the strings resulting from the splitting process are already proper parts of the original string, it follows that strings are gunky, and so no string is mereologically simple (let alone extended and simple).

While we are sympathetic to Baker's diagnosis, following Huggett \& Norton (2018) we note that it relies on a straightforward but particular interpretation of string theory, namely that splitting is a process in which a single string is divided into parts. It may be, however, that the process of splitting is really the annihilation of one string, followed by the creation of two new strings that bear no mereological connection to the original string. Thus, string theory doesn't exactly settle the matter. The simple material argument fails, then, if we don't supplement it with a further assumption about the nature of string splitting that rules out Baker's interpretation.

LQG does not fare much better. The trouble is that LQG is a theory only of the gravitational field. LQG does not include matter fields. Maybe they can be added to the theory, in which case there might be some reason to suppose it posits material entities. But until then, a realist attitude toward the theory does not carry any specific commitments to extended material objects. It certainly does not carry the same commitment as string theory, which clearly does posit a range of material entities (strings). One might respond that while LQG does not posit material objects yet, that is because it is not a final theory of QG. It is, at best, a step along the road to a final theory, and the final theory will include matter fields, and thus will include material objects. Perhaps this is right. Perhaps a suitably sophisticated 
version of the theory that has matter fields will posit extended simple material objects. To this we can only say: show us the theory.

We also have reason to doubt the object form of the harmony argument for both approaches to QG under consideration. This is straightforward for LQG (again, no matter, no deal). And for string theory: the most likely targets for the object form of the harmony argument in string theory are strings (or string-like objects, such as branes), and (as noted) it is controversial as to whether they are simple due to string-splitting. Moreover, harmony considerations don't seem to alleviate the controversy.

This leaves us with the simple region argument and the region version of the harmony argument. Note that the existence of spacetime, while necessary for these arguments to succeed, is not sufficient for their success. In addition to the existence of spacetime, we need one of two things. In the case of the simple argument, we need it to be the case that either string theory or LQG posits spatiotemporal regions that lack any parts. As noted in $\S 2$, in the case of the harmony argument, we also need the $\operatorname{discreteness}_{R}$ of spacetime. For if

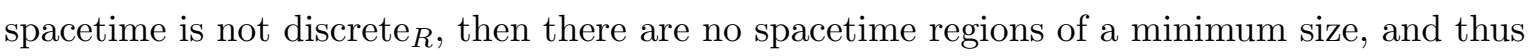
premise three in the harmony argument for regions is false.

Now, in so far as string theory might be thought to assume spacetime as a basic posit, the spacetime being assumed is made of points, not regions of a minimum size, and the points are topologically continuous (i.e. not isolated in the sense of $\operatorname{discreteness}_{T}$ ). There is little indication that spacetime will be discrete $_{R}$ even if it is posited by the theory (Baker 2016: $\S 4$ makes this point in detail). Thus, the region form of the harmony argument fails. The simple region argument succeeds only if string theory, under this interpretation, says of some (continuous) spacetime region that it lacks proper parts. So far as we can tell, there is no basis for attributing this claim to string theory, and so the simple argument for regions fails as well. 
In the case of LQG, by contrast, the existence of a spacetime that is discrete in some sense is a bit more plausible. While, as noted, it is difficult to link the spin-network structure to spacetime, it may in fact be that the nodes and edges in the spin-foam model represent aspects of a spatiotemporal structure. Since the spin-network structure is discrete, this suggests that the spacetime structure it represents is discrete as well. This opens the door to applying the two region arguments (simple and harmony) directly to the discrete spatiotemporal structure that a spin-network may represent.

But, in light of the distinction between discreteness $_{T}$ and discreteness $_{R}$ drawn above, we have to be careful here. For while it is commonly claimed that spin-networks in LQG are discrete, it remains unclear whether, when interpreted in spatiotemporal terms, the spin-

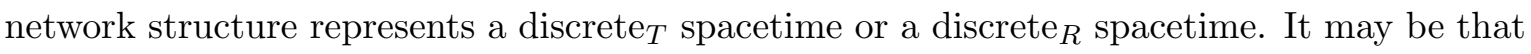
the nodes and edges in a spin-network represent quanta of spacetime - regions of a minimal size. Equally, however, the structure may correspond to a network of isolated spacetime points. So far as we can tell, the spin-network representation doesn't yet settle the matter one way or another.

But let us suppose, for the sake of argument, that the spin-network structure in LQG not only represents spacetime, but that it also entails that spacetime is $\operatorname{discrete}_{R}$ and thus that the nodes and edges of the spin-network structure represent spacetime regions of a minimum size. Given these assumptions, can a case for extended simples be made?

Focus, for a moment, on the simple region argument. This argument works only if the nodes and edges of spin-networks lack proper parts. For then a case can be potentially made that the extended regions of spacetime that correspond to those nodes and edges will be simple regions. The trouble is that the dynamical form of LQG - the spin-foam approachincludes operations for splitting and joining nodes and edges (see Magliaro \& Perini 2012 for discussion). Even in the kinematical form, Rovelli describes the way in which nodes and 
edges - what he calls 'grains of space' - can split to form other nodes and edges and thus other grains (see Grot \& Rovelli 1997; Rovelli 2011).

As in the string theory case, if the nodes and edges of a spin-network can be split, then it is unclear whether they are mereologically simple. It really depends on what node and edge splitting amounts to in LQG. It may be that the so-called 'process of splitting' of a node, say, is really the annihilation of this node, followed by the creation of two new nodes that bear no mereological connection to the original — or, to avoid reference to the time-related notions of creation and annihilation, that the three considered nodes do not hold in any constitutive relation and are just three distinct connected entities. Equally, however, the process may result in the splitting of nodes into parts. LQG, as it stands, does not seem to tell us exactly how to interpret node splitting. Accordingly, a simple realist commitment to LQG does not seem sufficient to warrant a commitment to extended simples: more is required.

This brings us to the harmony argument. If we assume that spacetime in LQG is discrete $_{R}$ and we assume $(\mathrm{H})$ then it does seem to follow that there are extended simples in LQG. To see this assume that some nodes and edges of a spin-networks structure correspond to spatiotemporal regions of a minimal size. Now, assume, for reductio, that these regions have parts. If they have parts, then by $(\mathrm{H})$ they have proper sub-regions. But then they are not regions of a minimal size, because there are smaller regions (namely the proper sub-regions). So they cannot have parts after all.

Aside from requiring the controversial assumption that LQG represents a discrete space-

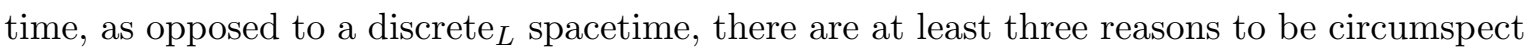
about the result of the harmony argument. First, as with the simple region argument, the harmony argument only works if node and edge splitting is assumed to be a process whereby new nodes are created, rather than as a case where nodes and edges are divided into parts. But, as we've already noted, it is unclear what to make of node and edge splitting in LQG. 
Second, the spin-foam approach is still a work in progress and so the matter remains unsettled (see e.g. Rovelli \& Vidotto 2014). Of particular interest is the fact that, in its dynamical form, LQG may not posit a discrete structure in any sense. This point has been made forcefully in Dittrich \& Thiemann (2009). If Dittrich and Thiemann are correct, however, and the spin-network structure is not discrete and so represents a continuous spacetime, then the harmony argument for regions fails for the same reason that it fails in the string theory case.

Third, and perhaps most importantly, full-blown realism - like spacetime eliminativismremains extremely controversial as an interpretation of, at least, LQG. The dominant interpretation of LQG among philosophers and among the physicists who developed the theory is that it denies the existence of spacetime in some sense. There is a real question, then, about whether a case for extended simples based on a full-blown realist interpretation of LQG does justice to the scientific understanding of that view. We recommend that philosophers of a naturalistic bent tread very carefully here.

In what remains, we will consider two further forms of spacetime realism that postulate the existence of spacetime only at some derivative levels of description. We will set string theory aside. For, as we saw, the arguments in favour of extended material objects for string theory are inconclusive. As we also saw, both the simple region argument and the harmony argument for simple regions fail in the string theory case. It is also unlikely that any of these arguments can be revived simply by considering different interpretations of spacetime realism. For in the case of material objects, the case for extended simples in string theory turns on whether strings can be split, not on how spacetime exists. In the case of extended simple regions, the existence of spacetime is relevant, but there is no evidence that string theory requires the existence of a discrete spacetime or of spacetime regions that lack parts. So, at the moment, there is no clear path from string theory to extended simples. We will 
also set aside the arguments in favour of simple material objects, since as we saw, LQG lacks matter. This leaves us with the two region arguments to consider for LQG.

\subsection{Mereological Spacetime Realism}

We come now to mereological spacetime realism. Mereological spacetime realism states that spacetime is composed of non-spatiotemporal entities. It is a member of a broader class of views - together with derivative spacetime realism to be discussed in the next sub-sectionaccording to which spacetime exists despite not being a basic posit of a theory of QG.

According to mereological spacetime realism, the nodes and edges of spin-networks do not represent a spatiotemporal structure. Instead, the nodes and edges in a spin-network are interpreted as non-spatiotemporal building blocks of spacetime. Because, on this interpretation, the spin-network structure is taken to describe the parts of spacetime, a realist commitment to LQG is taken to imply realism about spacetime as well via this mereological connection.

Given this, the simple argument for regions can be applied in two places. It can either be applied to the spin-network structure, or it can be applied to the spatiotemporal wholes that the spin-network structure composes. Neither option seems very appealing. The trouble with the first option is that even if the parts of spacetime are simple (and maybe they are) they are not spatiotemporally extended (though they may compose extended regions), so they are not extended simple regions. The trouble with the second option is that, according to mereological spacetime realism, all spatiotemporal regions have parts: they are composed of non-spatiotemporal building blocks. Either way, then, there is no obvious way to make a case for extended simples via realism about LQG alone.

The harmony argument also seems to fail. It fails because $(\mathrm{H})$ seems to be false: it is not the case that $x$ is part of $y$ iff $x$ 's location is a sub-region of $y$ 's location. Because the parts of 
spatiotemporal regions are not themselves located at any spatiotemporal region, they are not located at any sub-region of spacetime (assuming, as seems plausible, that spacetime regions have only other spatiotemporal regions as sub-regions).

The harmony argument can be revived, however. In order to see this, we need to consider mereological spacetime realism in more detail. Mereological spacetime realism can be developed by drawing on Paul's $(2002 ; 2012)$ approach to mereology. Paul differentiates between two kinds of mereological relations: spatiotemporal mereological relations and logical mereological relations. Whereas spatiotemporal mereological relations connect material objects, logical mereological relations (a new sui generis category of composition) relate properties and create objects. Consider the property of being a yellow circle. This property has two further properties as proper parts: the property of being a circle and the property of being yellow. Material objects are themselves regarded as conjunctions of properties including location properties. Consider the yellow chair in your room: it has the property of being yellow as one of its proper parts, and its location as another proper part. Thus parts are not always spatiotemporal in this approach, as spatiotemporal entities are themselves composed of non-spatiotemporal properties such as being yellow and location properties such as being located in your room.

On Paul's view, spacetime is built from more fundamental parts in the sense that spatiotemporal properties and relations are logically composite. Spatiotemporal mereology, however, bottoms out in spacetime: there are no mereological relations between spacetime and more fundamental non-spatiotemporal parts of the kind that relate entities within spacetime to one another, or that relate spacetime regions to one another.

Le Bihan (2018) develops a view that is similar to Paul's and applies it to the case of QG. Like Paul, Le Bihan also appeals to a non-spatiotemporal mereology. However, he does not endorse Paul's treatment of non-spatiotemporal mereology as a relation between proper- 
ties. Instead, he proposes to free non-spatiotemporal composition from the necessity to take properties as inputs and to give objects as outputs. He calls this sort of non-spatiotemporal composition 'trans-categorical composition' (Le Bihan 2018: 86). However, this name suggests that inputs and outputs must belong to distinct metaphysical categories, when (by his own lights) this need not be the case. As a generic way of referring to non-spatiotemporal mereology, we will refer to it instead as neutral mereology.

Spatiotemporal mereology involves the relationships between spatiotemporally located material objects contra neutral mereology. The thought, then, is that it is only spatiotemporal mereological relations to which harmony principles apply. On the view under consideration, harmony principles are not constraints on mereology more generally, and so don't apply to neutral mereology.

If that's right, then neutral mereology can be used to develop mereological spacetime realism as follows. First, spatiotemporally located objects are related by spatiotemporal mereological relations. These relations obey harmony principles, such as $(\mathrm{H})$. Second, spacetime itself is mereologically composed of more fundamental, non-spatiotemporal entities, specified by a theory of QG. These mereological relations are neutral mereological relations and don't obey $(\mathrm{H})$.

The distinction between neutral and spatiotemporal mereology presents an opportunity to differentiate between two ways of being simple. Consider, again, the definition of an extended simple material object introduced above. Given the distinction between neutral and spatiotemporal mereology, we can now differentiate between three varieties of simple region as follows:

[N-ESR] $x$ is a neutrally simple extended spatiotemporal region $=_{d f} x$ is an extended spatiotemporal region $r$ and $x$ has no proper neutral parts.

[S-ESR $] x$ is a spatiotemporally simple extended spatiotemporal region $=_{d f} x$ an 
extended spatiotemporal region $r$ and $x$ has no proper spatiotemporal parts.

[S\&N-ESR] $x$ is a neutrally and spatiotemporally simple extended spatiotemporal region $=_{d f} x$ is an extended spatiotemporal region $r$ and $x$ has no proper neutral or spatiotemporal parts.

Given this three-way distinction, an argument can be offered for the existence of extended simple regions by weakening $(\mathrm{H})$ so that it applies only to spatiotemporal mereology (call this weakened principle: $\left.\mathrm{H}^{*}\right)$ :

1. There exists at least one minimal extended spatiotemporal region $r$.

2. $r$ has a proper spatiotemporal part $p$ only if there exists a region $r *$ such that $r * \subset r$. $\left(\mathrm{H}^{*}\right)$

3. If $r$ is a minimal spatiotemporal region then there does not exist a spatiotemporal region $r *$ such that $r * \subset r$.

$\left(\right.$ Discreteness $\left._{R}\right)$

4. $r$ has no proper spatiotemporal parts.

5. $r$ is a spatiotemporally simple extended spatiotemporal region.

Call entities that satisfy S-ESR but not N-ESR 'complex simples' in order to underline their ambiguous nature with respect to the standard debates over extended simples. Complex simples are neutrally complex but spatiotemporally simple entities. What are we to say about ontologies that admit complex simples? Are complex simples extended simples in the strictest sense? It is largely up to us to decide. We could assume that complex simples are extended simples - thereby emphasising their spatiotemporal simplicity - or we could decide that complex simples differ too greatly from the standard notion of extended simples to categorize them as extended simples - thereby underlining their neutral complexity.

For the sake of argument, however, let us suppose that complex simples are extended simples. Under this supposition, mereological spacetime realism offers a potential path from 
physics to extended simples - understood as complex simples. But let's be clear: mereological spacetime realism is certainly not suggested by any research program to be found in contemporary physics. It is a metaphysical add-on. The physics alone does not provide evidence in favour of the existence of extended simples; it does so only within a certain metaphysical paradigm.

\subsection{Derivative Spacetime Realism}

The last realist option we consider is derivative spacetime realism. Derivative spacetime realism is another member of the class of broad views that take spacetime to exist, despite not being fundamental. Whereas mereological spacetime realism treats LQG as describing the parts of spacetime, the derivative spacetime realist interprets LQG as describing the grounds for spacetime, and thus appeals to a primitive grounding relation. Spacetime, on this view, is grounded in a range of non-spatiotemporal entities described by a theory of QG, and that's how it comes to exist.

As previously discussed, the notion of grounding that we have in mind is a primitive, metaphysical determination relation; one that is not be further specified via some other metaphysical relation. Because of the primitiveness of the grounding relation the view isn't very appealing. Contrast the grounding picture with the mereological account. The mereological account relieves at least some of the mystery of how spacetime exists. The existence of spacetime turns out to be very much like a style of mereological dependence of the nonfundamental on the fundamental found elsewhere in science. Derivative spacetime realism, by contrast, makes the existence of spacetime seem quite unlike more familiar pictures of non-fundamental ontology - thereby strengthening the conceptual gap between the spatiotemporal and the non-spatiotemporal.

At any rate, let's grant the view and look at its consequences for extended simples, 
starting with the simple region argument. As with mereological spacetime realism, derivative spacetime realism has consequences for realism about LQG more generally. Specifically, realism about LQG implies a commitment to not only the fundamental spin-network structure but to some non-fundamental spacetime structure as well via the grounding relation.

Now, we cannot draw on the fact that some nodes and edges of a spin-network have no parts to make a case for simple regions. These entities are not being interpreted as spatiotemporal regions. And so while they may be simple, they are not spatiotemporally extended. The only way for the simple region argument to work, then, is if the realist commitment to LQG carries with it a commitment to the existence of extended, simple spatiotemporal regions. It is difficult to see why this would be the case, however. Derivative spacetime realism doesn't bring with it specific commitments concerning what spacetime is like, only that there is some and that it's grounded in the fundamental structure described by LQG. Furthermore, the mereological nature of spacetime - namely, whether it bottoms out in extended simple regions or anything else - is not obviously settled by the nature of the ontology of the more fundamental level of description. So the mere fact of grounding does not seem to give us much insight into whether there are extended simple regions at the spacetime level.

This leaves us with the harmony argument. If we assume derivative spacetime realism and we further assume that spacetime is discrete $_{R}$, then the harmony argument seems to go through. That's because, under these assumptions, there are extended spacetime regions. Moreover, there are no smaller regions to be found at a more fundamental level. Finally, because the relationship between spacetime and a range of more fundamental, nonspatiotemporal entities is not mereological in nature there is no fear of falsifying $(\mathrm{H})$. There is thus no need for the weak harmony argument outlined in the previous section.

A case for extended simple regions based on a derivative realist approach to LQG can thus 
be made. Still, it is important to note that the case remains controversial. One must accept both the existence of a primitive grounding relation, and the discreteness $R$ of spacetime. While the second assumption may be borne out by further developments in physics, the first assumption appears to be a substantive metaphysical thesis, one that must be defended.

\section{Conclusion}

No doubt there are further ways to conceive of spacetime in the context of QG. We have not aimed to be exhaustive. We have, however, provided enough coverage to show how complex the case for extended simples really is. In the first instance, it is unclear whether theories like LQG and string theory posit spacetime enough for simples to exist. It is only when we couple those theories to a view on the existence of spacetime that a case for extended simples can be made. If we adopt an eliminativist approach to spacetime, then the case for extended simples is dead on arrival. Things are more promising if we adopt a realist approach to spacetime, but even there a great deal must be assumed in order to secure the existence of extended simples.

In general, we cannot hope to mount an argument for extended simples based on QG in isolation from a particular metaphysics of spacetime. At best one can mount a conditional argument: if the metaphysics of the physics is a certain way then the physics implies the existence of extended simples. But then it is not really the physics alone that implies the existence of extended simples (as a number of philosophers have suggested). It is, rather, some combination of physical and metaphysical assumptions on top of the physical results that is needed to make the case.

Sam Baron

Dianoia Institute of Philosophy, Australia 
Baptiste Le Bihan

University of Geneva, Switzerland

\section{Acknowledgements}

We are grateful to both reviewers for providing us with very helpful comments. The work of Baptiste Le Bihan was supported by the Swiss National Science Foundation. The work of Sam Baron was supported by the Australian Research Council through a Discovery Early Career Award (DE180100414) and a Discovery Project (DP180100105).

\section{References}

Baker, D. J. (2016) 'Does String Theory Posit Extended Simples?', Philosophers' Imprint, 16/18: $1-15$.

Baron, S. (2020) 'The Curious Case of Spacetime Emergence', Philosophical Studies, 177: $2207-226$.

Braddon-Mitchell, D. and K. Miller (2006) 'The Physics of Extended Simples', Analysis, 66/3: $222-26$.

Butterfield, J. (forthcoming) 'On Dualities and Equivalences Between Theories', in C. Wüthrich, B. Le Bihan and N. Huggett (eds.) Philosophy Beyond Spacetime. Oxford: Oxford University Press.

Butterfield, J. and C. Isham (1999) 'On the Emergence of Time in Quantum Gravity', in J. Butterfield (ed.) The Arguments of Time, 111-68. Oxford: Oxford University Press. 
Callender, C. (2011) 'Philosophy of Science and Metaphysics', in S. French and J. Saatsi (eds.) Continuum Companion to the Philosophy of Science, 33-54. London; New York: Continuum.

Crowther, K. (2018) 'Inter-theory Relations in Quantum Gravity: Correspondence, Reduction and Emergence', Studies in History and Philosophy of Modern Physics, 63: 74-85.

Crowther, K. and N. Linnemann (2019) 'Renormalizability, Fundamentality and a Final Theory: The Role of UV-Completion in the Search for Quantum Gravity', British Journal for the Philosophy of Science, 70/2: 377-406.

Dawid, R. (2013) String Theory and the Scientific Method. Cambridge: Cambridge University Press.

Dittrich, B. and T. Thiemann (2009) 'Are the Spectra of Geometrical Operators in Loop Quantum Gravity Really Discrete?', Journal of Mathematical Physics, 50/1: 012503.

Gilmore, C. (2018) 'Location and Mereology', in E. N. ZaIta (ed.) The Stanford Encyclopedia of Philosophy, Fall Edition, <https://plato.stanford.edu/archives/fall2018/entries/location-mereology/> accessed 03 March 2021.

Greene, B. (1999) The Elegant Universe: Superstrings, Hidden Dimensions, and the Quest for the Ultimate Theory. New York: Vintage.

Grot, N. and C. Rovelli (1997) 'Weave States in Loop Quantum Gravity', General Relativity and Gravitation, 29/8: 1039-1048.

Hořava, P. and E. Witten (1996) 'Heterotic and Type I String Dynamics from Eleven Dimensions', Nuclear Physics B, 460/3: 506-24. 
Huggett, N. (2017) 'Target Space $\neq$ = Space', Studies in History and Philosophy of Modern Physics, 59: 81-8.

Huggett, N. and J. Norton (manuscript) 'The Never-Ending Dancing of Philosophy and Physics'. Unpublished Draft.

Huggett, N. and T. Vistarini (2015) 'Deriving General Relativity from String Theory', Philosophy of Science, 82/5: 1163-74.

Huggett, N., Vistarini, T. and C. Wüthrich (2013) 'Time in Quantum Gravity', in A. Bardon and H. Dyke (eds.) A Companion to the Philosophy of Time, 242-261. London: WileyBlackwell.

Huggett, N. and C. Wüthrich (2013) 'Emergent Spacetime and Empirical (in)Coherence', Studies in History and Philosophy of Modern Physics, 44/3: 276-85.

Lam, V. and C. Wüthrich (2018) 'Spacetime is as Spacetime Does', Studies in History and Philosophy of Modern Physics, 64: 39-51.

Lam, V. and C. Wüthrich (forthcoming) 'Spacetime Functionalism from a Realist Perspective', Synthese.

Le Bihan, B. (2018) 'Space Emergence in Contemporary Physics: Why We Do Not Need Fundamentality, Layers of Reality, and Emergence', Disputatio, 49/10: 71-85.

Le Bihan, B. (2020) 'String Theory, Loop Quantum Gravity and Eternalism', European Journal for Philosophy of Science, 2/10: 1-22.

Le Bihan, B. and N. Linnemann (2019) 'Have We Lost Spacetime On the Way? Narrowing the Gap Between General Relativity and Quantum Gravity', Studies in History and Philosophy of Modern Physics, 65: 112-21. 
Le Bihan, B. and J. Read (2018) 'Duality and Ontology', Philosophy Compass, 13/12: e12555.

Magliaro, E. and C. Perini (2012) 'Local Spin Foams', International Journal of Modern Physics D, 21/13: 1-16.

Markopoulou, F. and L. Smolin (2007) 'Disordered Locality in Loop Quantum Gravity States', Classical and Quantum Gravity, 24/15: 3813.

Markosian, N. (2014) 'A Spatial Approach to Mereology', in S. Kleinshmidt (ed.) Mereology and Location, 69-90. Oxford: Oxford University Press.

McDaniel, K. (2007a) 'Extended Simples', Philosophical Studies, 133/1: 131-41.

McDaniel, K. (2007b) 'Brutal Simples', in D. W. Zimmerman (ed.) Oxford Studies in Metaphysics Volume 3, 233-66. Oxford: Oxford University Press.

Paul, L. A (2002) 'Logical Parts', Nô̂s, 36/4: 578-96.

Paul, L. A. (2012) 'Building the World from its Fundamental Constituents', 158/2: 221-56.

Polchinksi, J. (1998) 'String Theory: Volume 1, An Introduction to the Bosonic String'. Cambridge: Cambridge University Press.

Rettler, B. (2019) 'Mereological Nihilism and Puzzles About Material Objects', Pacific Philosophical Quarterly, 99/4: 842-68.

Rovelli, C. (2004) Quantum Gravity. Cambridge: Cambridge University Press.

Rovelli, C. (2011) 'A New Look at Loop Quantum Gravity', Classical Quantum Gravity, 28: $1-24$. 
Rovelli, C. and F. Vidotto (2014) Covariant Loop Quantum Gravity: An Elementary Introduction to Quantum Gravity and Spinfoam Theory. Cambridge: Cambridge University Press.

Saucedo, R. (2011) 'Parthood and Location', in D. W. Zimmerman and K. Bennett (eds.) Oxford Studies in Metaphysics Volume 5, 225-86. Oxford: Oxford University Press.

Strominger, A., Shing-Tung, Y. and E. Zaslow (1996) 'Mirror Symmetry is T-Duality', Nuclear Physics B, 479/1-2: 243-59.

Witten, E. (1996) 'Reflections on the Fate of Spacetime', Physics Today. 49/4: 24-31.

Wüthrich, C. (2017) 'Raiders of the Lost Spacetime', in D. Lehmkuhl, G. Schiemann and E. Scholz (eds.) Towards a Theory of Spacetime Theories, 297-335. Berlin: Springer.

Yates, D. (forthcoming) 'Thinking About Spacetime', in C. Wüthrich, B. Le Bihan and N. Huggett (eds.) Philosophy Beyond Spacetime. Oxford: Oxford University Press. 